\title{
A bacterial haloalkane dehalogenase gene as a negative selectable marker in Arabidopsis
}

\author{
Henrik Næsted", Marko Fennema ${ }^{2}$, Lin Hao ${ }^{1}$, \\ Mathias Andersen ${ }^{1}$, Dick B. Janssen ${ }^{2}$ and John Mundy ${ }^{1}{ }^{*}$ \\ ${ }^{1}$ Department of Plant Physiology, Molecular Biology \\ Institute, Copenhagen University, Øster Farimagsgade \\ 2A, 1353 Copenhagen K, Denmark, and \\ ${ }^{2}$ Department of Biochemistry, Groningen Biomolecular \\ Siences and Biotechology Institute, University of \\ Groningen, Nijenborgh 4, 9747 AG, Groningen, The \\ Netherlands
}

\section{Summary}

The dhIA gene of Xanthobacter autotrophicus GJ10 encodes a dehalogenase which hydrolyzes dihaloalkanes, such as 1,2-dichloroethane (DCE), to a halogenated alcohol and an inorganic halide (Janssen et al., 1994, Annu. Rev. Microbiol. 48, 163-191). In Xanthobacter, these alcohols are further catabolized by alcohol and aldehyde dehydrogenase activities, and by the product of the $d h / B$ gene to a second halide and a hydroxyacid. The intermediate halogenated alcohols and, in particular, the aldehydes are more toxic than the haloalkane substrates or the pathway products. We show here that plants, including Arabidopsis, tobacco, oil seed rape and rice, do not express detectable haloalkane dehalogenase activities, and that wild-type Arabidopsis grows in the presence of DCE. In contrast, DCE applied as a volatile can be used to select on plates or in soil transgenic Arabidopsis which express dhIA. The dhIA marker therefore provides haloalkane dehalogenase reporter activity and substrate dependent negative selection in transgenic plants.

\section{Introduction}

Negative selectable marker genes generally encode enzymes which metabolize a specific substrate to a cytotoxic product. For example, transgenic plants expressing $E$. coli $\operatorname{cod} A$, which deaminates 5-fluorocytosine (5-FC) to cytotoxic 5-fluorouracil, are unable to grow on media containing 5-FC (Stougaard, 1993). Such substrate-dependent negative markers can be used in genetic techniques including targeted recombination, tissue-specific cell

Received 14 January 1999; revised 16 April 1999; accepted 19 April 1999. *For correspondence (fax +4535322128; e-mail mundy@biobase.dk). death, and mutant screens. For example, Agrobacterium tms2, which hydrolyzes indole-3-acetamide to the auxin indole-3-acetic acid, is used as a marker in a maize $D s$ transposon system to select for transposition events that are unlinked to the donor loci (Sundaresan etal., 1995). Expression in tapetal cells of $S$. griseolus $\mathrm{P}^{450}$ su1, which converts the sufonylurea R70402 to a potent herbicide, can be used to produce male sterility (O'Keefe etal., 1994). A constitutively expressed nitrate reductase gene can be used in chlorate resistant selections of mutants impaired in nitrogen uptake, metabolism or their regulation (Nussaume et al., 1991). Lastly, Arabidopsis alcohol dehydrogenase, which hydrolyzes allyl alcohol to the toxic aldehyde acrolein, has been used in a fusion genetic screen to select trans-acting regulatory mutants (LópezJuez et al., 1998).

A novel substrate-dependent negative selection marker based on the $d h / A$ gene of Xanthobacter autotrophicus GJ10 is presented here. The $d h I A$ gene encodes a single chain, co-factor independent dehalogenase which hydrolyzes haloalkanes, such as 1,2-dichloroethane (DCE), to a halogenated alcohol and an inorganic halide (Janssen et al., 1994). The X-ray structure and catalytic mechanism of the enzyme have been determined (Schanstra etal., 1996; Verschueren etal., 1993) In Xanthobacter, the alcohols produced are further catabolized by alcohol and aldehyde dehydrogenase (ADH and ALDH) activities, and by the $d h / B$ haloalkanoic acid dehalogenase to a second halide and a hydroxyacid (Figure 1). The intermediate halogenated alcohols and particularly the aldehydes are far more toxic than the haloalkane substrates or the final products of the pathway.

Two potential uses of the $d h / A$ encoded haloalkane dehalogenase (DhIA) can be envisioned in transgenic plants. First, because plant ADH and ALDH activities are normally low in roots and leaves (op den Camp and Kuhlemeier, 1997), plants expressing high levels of DhIA haloalkane dehalogenase and supplied with DCE or similar substrates might accumulate toxic levels of the product alcohols or aldehydes. In this case, and as shown here, $d h I A$ can be used as a substrate-dependent negative marker. Second, various DhIA substrates are important environmental pollutants in soil and groundwater, including the bulk synthetic chemical DCE and the fumigants dichloropropene and DBE. Transgenic plants capable of fully degrading such compounds via the activity of DhIA 
572 Henrik Næsted et al.

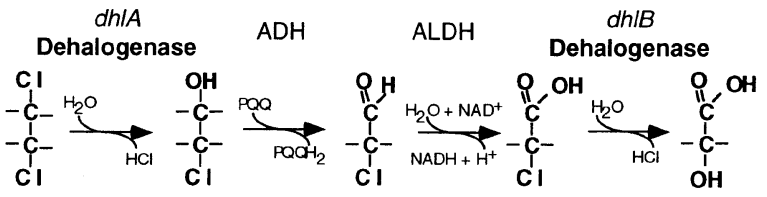

Figure 1. Catabolic pathway of 1,2-dichloroethane in $X$. autotrophicus (from Janssen et al., 1994).

$\mathrm{ADH}$, alcohol dehydrogenase; $\mathrm{ALDH}$, aldehyde dehydrogenase; $\mathrm{PQQ}$, $\mathrm{ADH}$ pyrroloquinoline quinone cofactor; NAD, ALDH nicotinamide adenine dinucleotide co-factor.

Single T-DNA locus Multiple T-DNA loci

wt $\longdiv { 2 4 5 1 7 2 4 2 5 2 7 2 8 3 0 3 7 8 1 1 1 2 1 5 2 0 2 2 2 3 2 6 }$

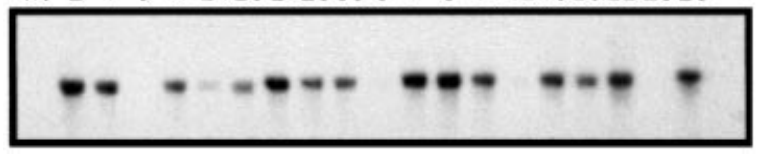

Figure 2. Northern blot analysis of dhIA RNA in Arabidopsis lines transformed with the $2 \times 35 \mathrm{~S} / d h / A$ construct.

$10 \mu \mathrm{g}$ total RNA was loaded per lane. Line numbers (top) are grouped as containing single or multiple T-DNA loci (T2 seed exhibiting 3:1 KAN ${ }^{R}$ or $\left.>3: 1 \mathrm{KAN}^{\mathrm{R}}\right)$. Wt. control is untransformed Ecotype Ws. The filter was exposed for $6 \mathrm{~h}$.

could therefore be useful in bioremediation of contaminated sites (Janssen et al., 1994). As a first step, we show here that DhIA dehalogenase can be expressed in Arabidopsis, and that the $d h / A$ gene can be used as a negative marker in plants.

\section{Results}

\section{Production of transgenic Arabidopsis expressing the} dhlA gene

The $d h / A$ coding region was isolated as a linkered PCR fragment from plasmid pKLA (Schanstra etal., 1993) and directionally cloned between the double CaMV 35S promoter and pea SSU E9 terminator in pKYLX71:35S2 (Schardl etal., 1987). The resulting construct was introduced into Arabidopsis and transgenic lines selected on kanamycin $\left(K_{A N}{ }^{R}\right.$ ) were allowed to self (Bechtold etal., 1993). KAN ${ }^{R} T 2$ plants among progeny exhibiting 3:1 KAN ${ }^{R}$ segregation (single integration site) or $>3: 1 K^{K A N^{R}}$ segregation (multiple insertions) were allowed to self-pollinate and the seed from T3 lines homozygous for at least one TDNA insertion locus ( $100 \% \mathrm{KAN}^{\mathrm{R}}$ progeny) was collected. No abnormal phenotypic effects under normal growth conditions were noted for any transgenic line.

PCR on leaf DNA (not shown) and Northern blotting were used to identify kanamycin-resistant transgenic lines expressing dhIA RNA (Figure 2). The levels of expression among lines containing single and multiple T-DNA loci
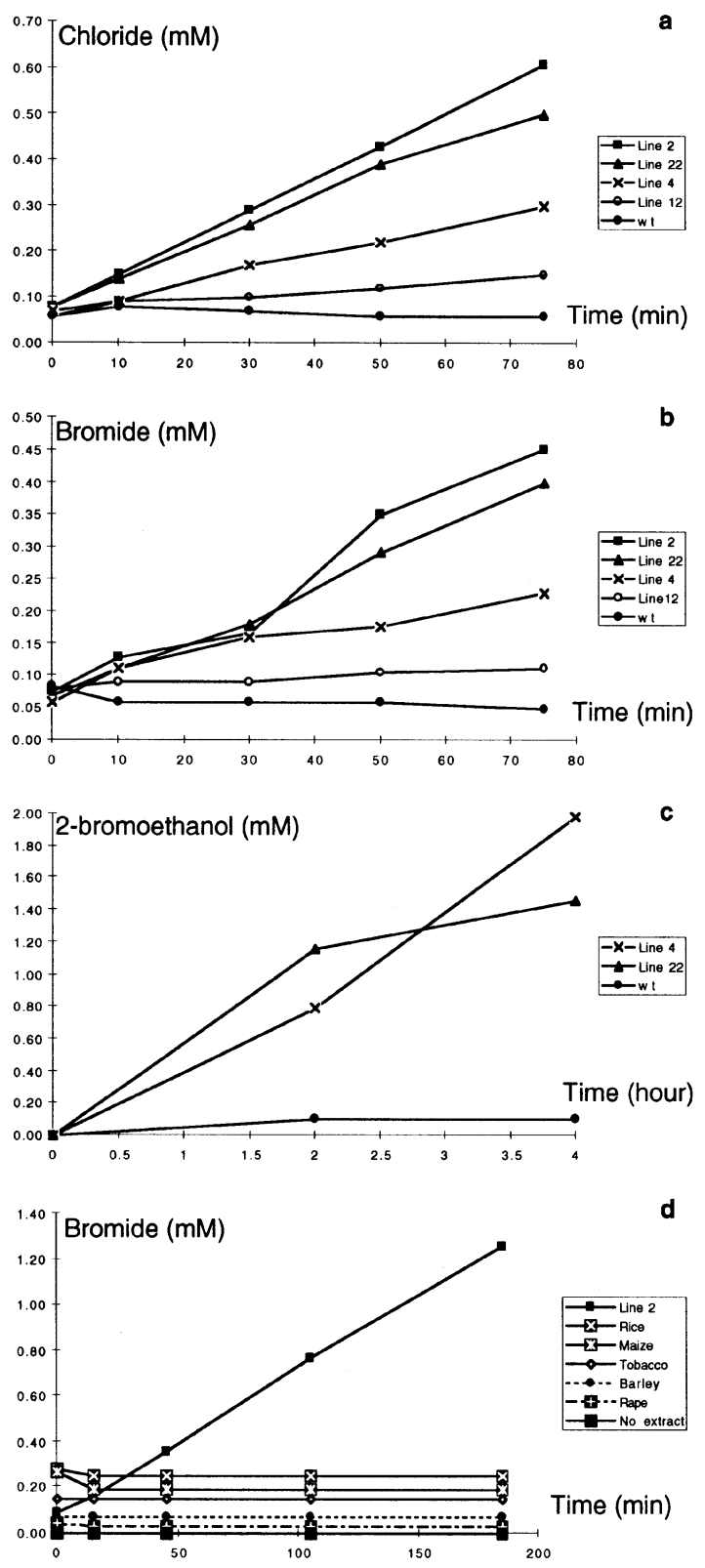

Figure 3. Haloalkane dehalogenase activities of transgenic Arabidopsis and other plants.

Halide production from (a) 1,2-dichloroethane and (b) 1,2-dibromoethane by extracts of wild-type and transgenic Arabidopsis lines as noted. (c) Production of 2-bromoethanol from 1,2-dibromoethane by Arabidopsis extracts. (d) Halide production from 1,2-dibromoethane by different plant species.

varied, presumably due to copy number and position effects.

Measurements of haloalkane dehalogenase activities in transgenic Arabidopsis and in other plants

Lines 2, 4, 12 and 22 accumulating relatively high levels of dhIA RNA were tested for their ability to dehalogenate the 
model substrates DCE and DBE (Figure 3a,b). Dehalogenase activity was detected in the transgenic lines but not in the untransformed control, and the levels of enzyme activity in the transgenic lines correlated well with the levels of $d h I A$ RNA detected in them by Northern blotting (Figure 2). These results demonstrate that active DhIA dehalogenase can be expressed in Arabidopsis.

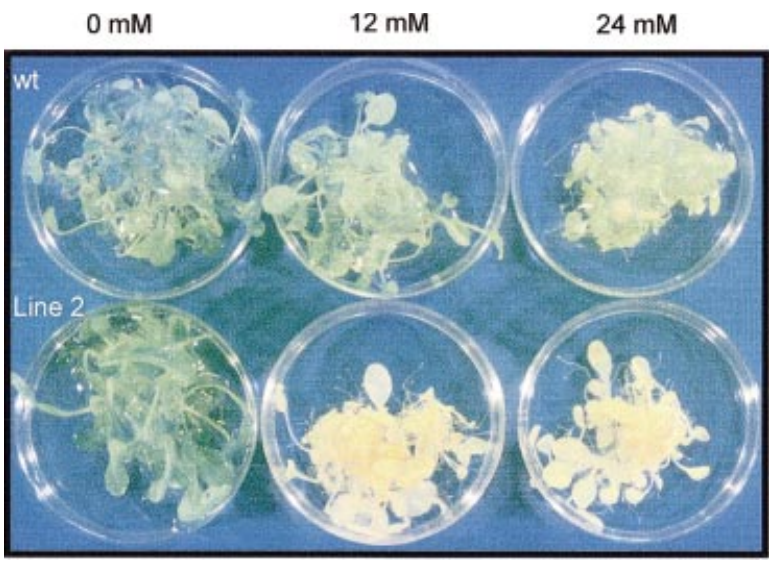

Figure 4. Negative selection using DCE.

Phenotypic effects of DCE added to liquid cultured, 2-week-old wild-type (upper row) or line $2 \mathrm{dh} / \mathrm{A}$ transgenic (lower row) plantlets for $48 \mathrm{~h}$.
Table 1 shows that the specific activities for DCE of extracts of lines 2, 4, 12 and 22 range from $34 \mathrm{mU}$ to $228 \mathrm{mU} \mathrm{mg}^{-1}$ extract protein. We calculate that in the highest expressing line 2, DhIA dehalogenase may represent $2 \%$ of total soluble extract protein, based upon the specific activity of the purified, recombinant enzyme expressed in E. coli (Schanstra etal., 1993). The ratio of the activities with DBE and DCE of the protein expressed in plants corresponds well with the activity ratios of the protein purified from E. coli. Most modifications of the protein result in a decrease of the ratio of DCE versus DBE

Table 1. Dehalogenating activities of transgenic Arabidopsis for 1,2-dibromoethane (DBE) and 1,2-dichloroethane (DCE)

\begin{tabular}{lll}
\hline Sample & $\begin{array}{l}\text { Spec. act. } \\
\text { for DCE } \\
\text { (mU } \mathrm{mg}^{-1} \text { protein) }\end{array}$ & $\begin{array}{l}\text { Spec.act. } \\
\text { for DBE } \\
\left(\mathrm{mU} \mathrm{mg} \mathrm{mg}^{-1} \text { protein) }\right.\end{array}$ \\
\hline Wild type & $<5$ & $<5$ \\
2 & 228 & 175 \\
4 & 119 & 77 \\
12 & 34 & 13 \\
22 & 188 & 140 \\
\hline
\end{tabular}
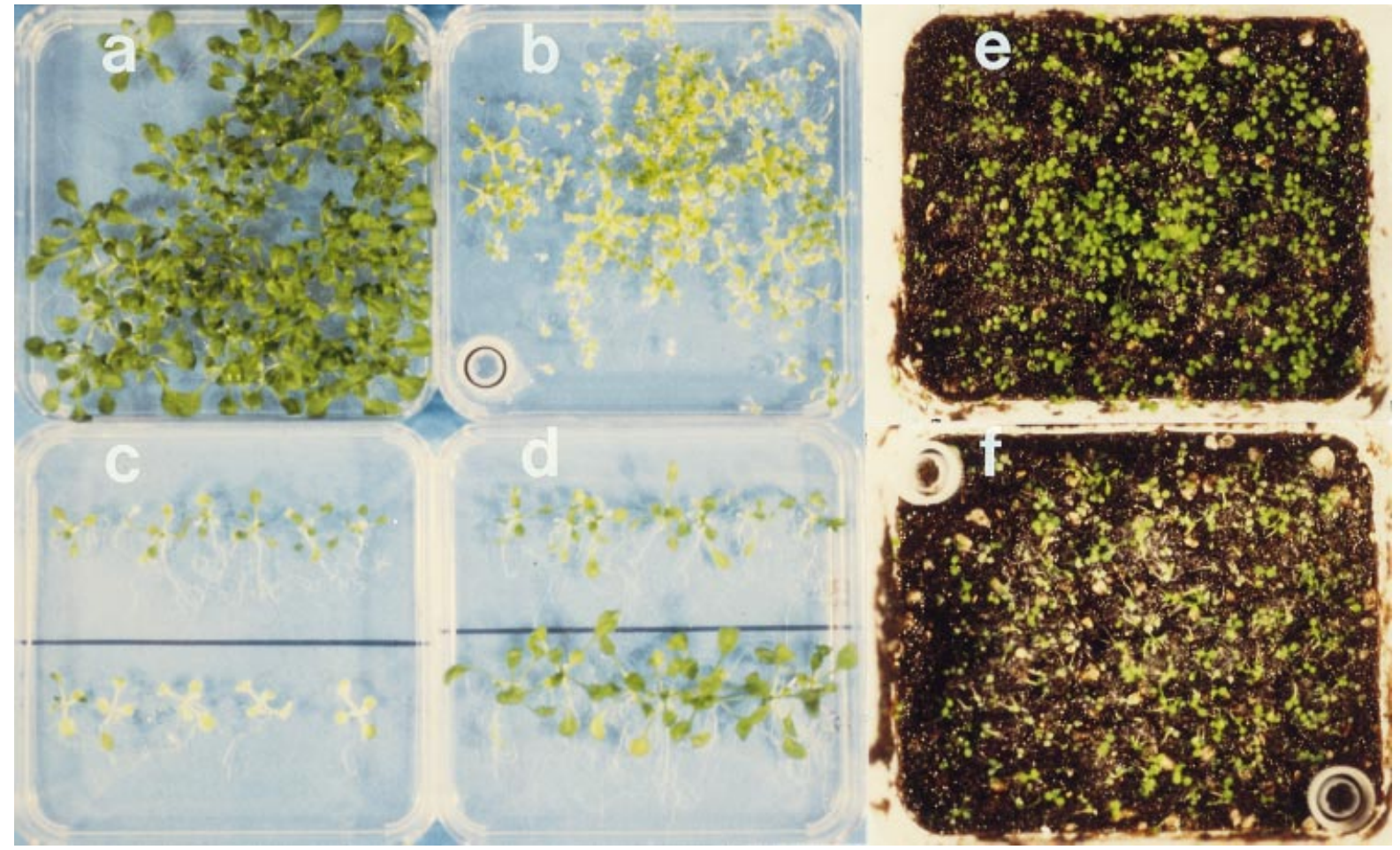

Figure 5. Reconstitution screen for negative selection using DCE.

(a) Phenotype after 16 days of growth on MS agar plates of a 1:25 mixture of untransformed, wild-type seed and seed of transgenic line 2. (b) The same mixture after 14 days of growth followed by 2 days of selection in the presence of $5 \mu$ l of volatile DCE added to a microtube cap. Plantlets selected in (b) as transgenic (upper row) or wild-type (lower row) were transferred to MS/Kan (c) or MS (d) plates and allowed to grow for an additional 6 days. Phenotypes of a 1:10 mixture of wild-type and transgenic line 2 seeds after 16 days in soil (e) and 14 days in soil followed 2 days of selection with $30 \mu$ of DCE added to a microtube cap. 
activity (Schanstra etal., 1996). The fact that this ratio is roughly the same in Arabidopsis indicates that the protein is correctly folded and not deleteriously modified.

The formation of 2-bromoethanol from 1,2-dibromoethane was also followed in the high expressing lines 4 and 22 (Figure 3c). Rapid production of 2-bromoethanol was found which indicates that the expected hydrolytic dehalogenation occurs in these plants.

Extracts of Arabidopsis, maize, rape, tobacco and barley were tested for endogenous activities towards DBE (Figure 3d) and DCE (not shown). None of these extracts showed significant levels of activity on either substrate, indicating that there is normally no haloalkane dehalogenating activity present in these plants.

Negative selection using 1,2-dichloroethane of transgenic Arabidopsis expressing dhIA

The effect of different concentrations of DCE on the growth phenotypes of wild-type and $d h / A$ expressing transgenic Arabidopsis was then examined. This showed that $12 \mathrm{~mm}$ DCE did not significantly effect the growth in liquid media of wild-type (Figure 4 upper) or plants transformed with an empty vector control (not shown). In contrast, this concentration of DCE caused severe bleaching of $d h / A$ transgenic lines 2 and 12 (Figure 4 lower).

To examine whether the $d h / A$ transgene could be used as a selectable marker in practical screening experiments, reconstitution assays were performed on plates and in soil with mixtures of wild-type and $d h I A$ expressing transgenic plantlets. This showed that low concentrations of DCE (approximately $1.5-2.0 \mathrm{~mm}$ in air), added in a microtube cap as a volatile liquid to MS plates or phytatrays with soil, did not significantly effect the growth phenotype of wildtype plantlets but led to severe bleaching of $d h / A$ transgenic plantlets (Figure $5 \mathrm{a}$ versus $5 \mathrm{~b}$ and $5 \mathrm{e}$ versus 5f). The efficacy of the selection was demonstrated by transferring plantlets scored phenotypically as transgenic or wild-type to MS/KAN or MS plates (Figure $5 \mathrm{c}, \mathrm{d}$ ). This showed that plants selected as $d h / A$ transgenics recovered normal growth on both MS/KAN and MS (Figure 5c,d, top row), while plants selected as wild-type grew well on MS but not on MS/KAN (Figure $5 c$ versus $5 d$, lower row). In three experiments, only two out of $300 \quad(<1 \%)$ plants scored as DCE resistant proved to be not kanamycin resistant upon transfer to antibiotic plates and were therefore escapes.

\section{Discussion}

Expression of the bacterial DhIA dehalogenase from a constitutive promoter in Arabidopsis provides negative selection with millimolar concentrations of DCE. This selection system works well for plants growing both on plates and in soil. It is amenable to large scale screening because the DCE substrate is volatile and can be easily added in small volumes to closed containers at selected times during the growth of plants. Recovery of selected $d h / A$ expressing transgenic plants is also rapid following transfer after DCE selection.

While comparable, the use of $d h / A$ as a negative marker may have three advantages over the use of alcohol dehydrogenase (ADH). First, negative selection on allyl alcohol of plants expressing an $\mathrm{ADH}$ transgene requires the use of an ADH null allele background for transformation (López-Juez et al., 1998). This limitation may complicate subsequent genetic analyses such as mapping studies. Because most plants do not express detectable haloalkane dehalogenase activity, the action of a $d h / A$ transgene is not masked by an endogenous activity. As shown here, this should be true for a wide variety of plants other than Arabidopsis. Second, DCE is considerably less toxic than allyl alcohol, which may comfort users (Sax, 1984). However, both the aldehydes derived from DCE (2chloroacetaldehyde) and allyl alcohol (acrolein) are highly reactive, their catabolites potentially mutagenic, and the accumulation of either may be equally toxic. Third, practical screening with $d h / A$ does not require substrate selection in liquid culture, as may be the case for ADH (López-Juez et al., 1998). It should be noted that, as for any marker-based, fusion genetic screen, the choice of a transgenic line expressing dhIA under the control of a promoter of interest, as well as the conditions for selection (substrate concentration and time course of incubation), must be empirically determined.

A question remains as to the exact mechanism of negative selection in plants with $d h I A$. By analogy to other systems, chloroacetaldehyde, derived from DCE via transgenic DhIA and endogenous ADH activities, is probably the major toxic catabolite produced (Janssen et al., 1994). In keeping with this, we found that growth of $d h / A$ transgenic plants is inhibited on chloroethanol (data not shown), perhaps due to the formation of chloroacetaldehyde via endogenous ADH. To directly address this question, we examined the growth in liquid culture of wild-type and $\mathrm{ADH}$ null plants in the absence and presence of $12 \mathrm{~mm}$ chloroethanol or allyl alcohol as a control (Dolferus et al., 1990; López-Juez et al., 1998). This concentration of substrates produced no detectable effects on plant growth after 2 days of incubation, in contrast to the toxic effect of the same concentration of DCE on transgenic dhIA plants (Figure 3). Continued incubation in chloroethanol for 14 days produced complete bleaching of wild-type, in contrast to $A D H$ null plants which exhibited bleached leaves but green leaf bases, stems and meristem (data not shown). These symptoms are similar to those of dhIA transgenic plants treated briefly with DCE, as could be 
seen microscopically prior to transfer in the reconstitution experiments (Figure 5).

This indicates that chloroacetaldehyde is the major toxic metabolite produced from DCE in dhIA transgenic plants, as suggested by the relative toxicities of DCE metabolites in animals (Sax, 1984). It also suggests that the effect of transgenic dhIA activity is cell autonomous, in that systemic cell death did not appear to occur in ADH null plants treated with chloroethanol or in dhIA transgenic plants under selection with DCE.

These results suggest that engineered expression of downstream aldehyde dehydrogenase (op den Camp and Kuhlemeier, 1997) and $d h / B$ haloalkanoic acid dehalogenase, or of other detoxification pathways (Martinola etal., 1993), might lead to the development of bioremediating plants capable of degrading DCE and related haloalkane pollutants. In any event, constitutive or regulated expression of $d h / A$ may be used as a substrate-dependent negative marker in plants.

\section{Experimental procedures}

\section{Construction of the dhIA marker and analyses of transgenic plants}

The $d h I A$ coding region was amplified from pKLA (Schanstra et al., 1993) by PCR with Vent polymerase (New England Biolabs) using a $5^{\prime}$ Xhol linker-primer (CTCGAGCCATGGTAAATGCAATTCGCACC, start MET bold) and a $3^{\prime}$ Xbal linker-primer (CTCAGACTCTATTCTGTCTCGGCAAAGTG, stop bold). Following digestion with $\mathrm{Xhol} / \mathrm{Xbal}$, the fragment was directionally cloned in these sites as a transcriptional fusion in pKYLX71:35S2 (Schardl et al., 1987). The plasmid was transferred by electroporation to Agrobacterium strain PGV3101 which was used to transform Arabidopsis ecotype Ws by vacuum infiltration (Bechtold et al., 1993). $\operatorname{Kan}^{R}$ plantlets were selected by standard procedures and leaf DNA for PCR with $d h / A$-specific primers and total RNA for Northern analysis were isolated by mini-procedures (Klimyuk etal., 1993; Nagy etal., 1988). $10 \mu \mathrm{g}$ total RNA per lane were separated in formaldehyde gels, blotted to Hybond $\mathrm{N}$ nylon filters and probed with $d h / A$ coding sequences ${ }^{32} \mathrm{P}$-labelled by nick translation after standard protocols.

\section{Dehalogenase assays}

Dehalogenase activity was determined by the release of halide in a colorimetric assay. $50 \mu \mathrm{l}$ of extract was incubated with $3 \mathrm{ml}$ of $5 \mathrm{mM}$ substrate solution in $50 \mathrm{mM}$ Tris buffer $(\mathrm{pH} 8.2)$ at $30^{\circ} \mathrm{C}$. Halide production in $0.5 \mathrm{ml}$ samples was determined spectrophotometrically at $460 \mathrm{~nm}$ with mercuric thiocyanate and ferric ammonium sulphate (Bergmann and Sanik, 1957). One activity unit was defined as the amount of enzyme that catalysed the formation of $1 \mu \mathrm{mol}$ halide per minute.

The formation of the product 2-bromoethanol was analyzed by gas chromatography. A $0.5 \mathrm{ml}$ sample was removed from the incubation mixture and extracted with $2 \mathrm{ml}$ diethylether containing $0.05 \mathrm{~mm}$ 1-bromohexane as an internal standard. The diethylether fraction was injected on a Chrompack 483 gas chromotograph (Chrompack, Middelburg, the Netherlands) fitted with an electron capture detector. The separation of substrate and product was undertaken on a Chrompack CPWax 52 column with the following temperature program: $3 \mathrm{~min}$ isothermal at $60^{\circ} \mathrm{C}$ followed by an increase of $10^{\circ} \mathrm{C} / \mathrm{min}$ to $240^{\circ} \mathrm{C}$, and ending with an isothermal segment of $5 \mathrm{~min}$ at $240^{\circ} \mathrm{C}$.

\section{Negative selection with 1,2-dichloroethane}

Seeds and mixtures of wild-type and transgenic seeds were surface sterilized and plated on MS media without or with $50 \mu \mathrm{g} \mathrm{ml}^{-1}$ kanamycin after autoclaving, or sown as a sand mix directly on $2-3 \mathrm{~cm}$ of autoclaved soil in a phytatray (Sigma). After cold treatment for 2 days at $4^{\circ} \mathrm{C}$, plants were grown under $16 \mathrm{~h}$ light at $21^{\circ} \mathrm{C}$. The effect of different DCE concentrations on the growth of wild-type and $d h / A$ transgenics was initially examined after transfer of 10 -day-old plants to liquid $0.5 \times$ MS for 4 days followed by $48 \mathrm{~h}$ treatment with DCE added to the media. For reconstitution experiments, volatile, liquid DCE in a microtube cap was placed in parafilm-sealed plates $(5 \mu \mathrm{l})$ or phytatrays $(30 \mu \mathrm{l})$ for selection of 10-day-old plants for 2 days under constant light. These liquid DCE volumes fully volatilize and correspond to approximately $1.5-2.0 \mathrm{mM}$ DCE in air. Following selection on plates, plants were rescued by transfer to either MS or MS/KAN plates and fully recovered after 6-8 days of growth.

\section{References}

Bechtold, N., Ellis, J. and Pelletier, G.X. (1993) In planta Agrobacterium mediated gene transfer by infiltration of adult Arabidopsis plants. C.R. Acad. Sci. Paris. Life Sci. 316, 11941199.

Bergmann, J.G. and Sanik, J. (1957) Determination of trace amounts of chloride in naphtha. Anal. Chem. 29, 241-243.

op den Camp, R.G.L. and Kuhlemeier, C. (1997) Aldehyde dehydrogenase in tobacco pollen. Plant Mo. Biol. 35, 355-365.

Dolferus, R., Van Den Bossche, D. and Jacobs, M. (1990) Sequence analysis of two null-mutant alleles of the single Arabidopsis Adh locus. Mol. Gen. Genet. 224, 297-302.

Janssen, D.B., Pries, F. and van der Ploeg, J.R. (1994) Genetics and biochemistry of dehalogenating enzymes. Annu. Rev. Microbiol. 48, 163-191.

Klimyuk, V.I., Carroll, B.J., Thomas, C.M. and Jones, J.D. (1993) Alkali treatment for rapid preparation of plant material for reliable PCR analysis. Plant J. 3, 493-494.

López-Juez, E., Jarvis, R.P., Takeuchi, A., Page, A.M. and Chory, J. (1998) New Arabidopsis cue mutants suggest a close connection between plastid- and phytochrome regulation of nuclear gene expression. Plant Physiol. 118, 803-815.

Martinola, E., Grill, E., Tommasini, R., Kreuz, K. and Amrhein, N. (1993) ATP-dependent glutathione S-conjugate 'export' pump in the vacuolar membrane of plants. Nature, 364, 247-249.

Nagy, F., Kay, S.A. and Chua, N.-H. (1988) Analysis of gene expression in transgenic plants. In Plant Molecular Biology Manual (Gelvin, S.B. and Schilperoort, R.A., eds). Dordrecht: Kluwer Academic Publishers, 1-29.

Nussaume, L., Vincentz, M. and Caboche, M. (1991) Constitutive nitrate reductase: a dominant conditional marker for plant genetics. Plant J. 1, 267-274.

O'Keefe, D.P., Tepperman, J.M., Dean, C., Leto, K.J., Erbes, D.L. and Odell, J.T. (1994) Plant expression of a bacterial cytochrome P450 that catalyzes activation of a sulfonylurea Pro-herbicide. Plant Physiol. 105, 473-482. 


\section{Henrik Næsted et al.}

Sax, N.I. (1984) Dangerous Properties of Industrial Materials. 6th edn. Van Nostrand Reinhold Co.

Schanstra, J.P., Rick, R., Pries, F. and Janssen, D.B. (1993) Construction of an expression and site-directed mutagenesis system of haloalkane dehalogenase in Eschericia coli. Protein Expr. Purif. 4, 479-489.

Schanstra, J.P., Ridder, I.S., Heimeriks, G.J., Rink, R., Poelarends, G.J., Kalk, K.H., Dijkstra, B.W. and Janssen, D.B. (1996) Kinetic characterization and $\mathrm{X}$-ray structure of a mutant of haloalkane dehalogenase with higher catalytic activity and modified substrate range. Biochemistry, 35, 13186-13195.

Schardl, C., Byrd, A.D., Benzion, G.B., Altschuler, M.A., Hildebrand, D.F. and Hunt, A.G. (1987). Design and construction of a versatile system for the expression of foreign genes in plants. Gene, 61, 1-11.

Stougaard, J. (1993) Substate-dependent negative selection in plants using a bacterial cytosine deaminase gene. Plant J. 3, 755-761.

Sundaresan, V., Springer, P., Volpe, T., Haward, S., Jones, J.D.G., Dean, C., Ma, H. and Martienssen, R. (1995) Patterns of gene action in plant development revealed by enhancer trap and gene trap transposable elements. Genes Dev. 9, 1797-1810.

Verschueren, K.H., Seljee, F., Rozeboom, H.J., Kalk, K.H. and Dijkstra, B.W. (1993) Chrystallographic analysis of the catalytic mechanism of haloalkane dehalogenase. Nature, 363, 693-698. 